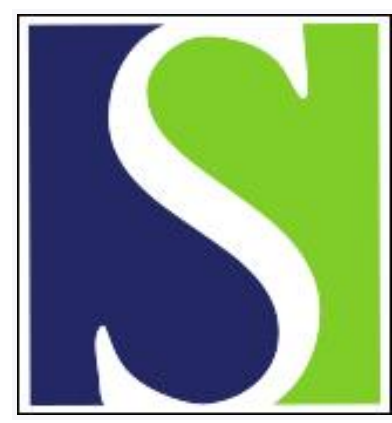

Scand J Work Environ Health Online-first -article

https://doi.org/10.5271/sjweh.2896

Published online: 26 Jan 2010

Mortality from circulatory system diseases and low-level radon exposure in the French cohort study of uranium miners, 1946-1999

by Nusinovici S, Vacquier B, Leuraud K, Metz-Flamant C, Caër-Lorho S, Acker A, Laurier D

Affiliation: Institut de Radioprotection et de Sûreté Nucléaire, DRPH/SRBE/LEPID, BP17, 92262 Fontenay aux Roses, France. simon.nusinovici@irsn.fr

Refers to the following texts of the Journal: 1997;23(3):221-226 1989;15(3):165-179

Key terms: cardiovascular disease; cerebrovascular disease; CeVD; circulatory system disease; cohort study; CVD; exposure; France; ionizing radiation; low-level exposure; low-level radon exposure; miner; mortality; occupational exposure; radiation; radon exposure; uranium miner

This article in PubMed: www.ncbi.nlm.nih.gov/pubmed/20101380 


\title{
Mortality from circulatory system diseases and low-level radon exposure in the French cohort study of uranium miners, 1946-1999
}

\author{
by Simon Nusinovici, MSc, ${ }^{1}$ Blandine Vacquier, PhD, ${ }^{1}$ Klervi Leuraud, PhD, ${ }^{1}$ Camille Metz-Flamant, MSc, ${ }^{1}$ \\ Sylvaine Caër-Lorho, MSc, ${ }^{1}$ Alain Acker, MD, ${ }^{2}$ Dominique Laurier, $P h D^{1}$
}

Nusinovici S, Vacquier B, Leuraud K, Metz-Flamant C, Caër-Lorho S, Acker A, Laurier D. Mortality from circulatory system diseases and low-level radon exposure in the French cohort study of uranium miners, 1946-1999. Scand J Work Environ Health. 2010;36(5):373-383.

Objectives The few studies examining the risk of circulatory system diseases (CSD) associated with ionizing radiation have reported inconsistent results. Radon, a known pulmonary carcinogen, emits ionizing radiation. The aim of this study was to examine CSD mortality in a French cohort of uranium miners and evaluate the plausibility of an association with radon exposure.

Methods The cohort included men employed as uranium miners for $\geq 1$ year between 1946-1990. We obtained vital status and cause of death from national registers and reconstructed radon exposure for each year. Exposurerisk relations were estimated with a linear excess relative risk (ERR) model using a 5-year lag time.

Results The cohort comprised 5086 miners, followed up for a mean duration of 30.1 years. The average cumulative exposure of the radon-exposed miners was 36.6 working level months (WLM). A total of 1411 deaths were observed, including 319 deaths due to CSD. No excess risk was found for this overall cause of death. A significant positive trend was observed between deaths from cerebrovascular diseases (CeVD) and cumulative radon exposure, together with a significant ERR per 100 WLM [ERR per 100 WLM $0.49,95 \%$ confidence interval $(95 \% \mathrm{CI})$ 0.07-1.23)]. Hard physical activity was identified as a potential modifying factor of the exposure-risk relation.

Conclusions For the first time in a cohort of uranium miners, our results suggest an association between CeVD mortality and cumulative radon exposure. Due to a lack of data, which limited our ability to assess possible confounding by cardiovascular risk factors, these findings should be interpreted with caution.

Key terms cardiovascular disease; CVD; cerebrovascular disease; CeVD; ionizing radiation; occupational exposure.

It has been postulated that exposure to high doses of external ionizing radiation may lead to the premature development of atherosclerosis and consequently to an increased risk of coronary heart disease. Evidence for this hypothesis has come from animal experiments $(1,2)$, the excess number of coronary heart disease deaths among women treated with radiation for breast cancer (3), and clinical reports of patients treated with radiotherapy who developed premature atherosclerosis thereafter (4). Very high doses of radiation to the heart can result in fibrotic and small vessel damage in the pericardium, myocardium and coronary vasculature, and subsequent myocardial infarction is the most common fatal complication (5).

At low doses of external ionizing radiation, the potential effects of radiation on non-cancer diseases have been reported only occasionally. The first evidence of an increased risk of circulatory system diseases (CSD) associated with low doses of external ionizing radiation came from an analysis of mortality data in the lifespan cohort study of Japanese atomic bomb survivors (6). This study observed an association with external dose for both coronary heart diseases and cerebrovascular diseases (CeVD). McGale \& Darby (7) reviewed all epidemiological studies reporting results about associations between low doses of ionizing radiation and CSD. Of 26 identified studies, only one - concerning US radiology technologists - provided appreciable evidence of an association between cumulative radiation exposure and CSD (8). Other evidence that low doses of external ionizing radiation are associated with an increased risk of CSD has come from a cohort

1 Institut de Radioprotection et de Sûreté Nucléaire, Fontenay aux Roses, France.

2 AREVA NC, Coordination Médicale, Paris, France.

Correspondence to: Dominique Laurier, Institut de Radioprotection et de Sûreté Nucléaire, DRPH/SRBE/LEPID, BP17, 92262 Fontenay aux Roses, France. [E-mail: dominique.laurier@irsn.fr] 
of international nuclear workers (9) and a recent study of Chernobyl clean-up workers (10). These results have not, however, shown a consistent pattern of increased mortality from CSD. More recently, other literature reviews confirmed this statement (11-13) indicating that the current knowledge is insufficient to support convincingly a causal interpretation of the observed associations.

Regarding the effects of internal contaminations, the available literature providing information on a potential risk of CSD is even more limited. Uranium miners constitute an interesting study population in this respect, as they are generally exposed to high levels of radon gas concentration. This radioactive gas could be inhaled and lead to internal irradiation due to the alpha emitters present amongst its decay products. Since the beginning of the 1980s, many studies of uranium miners cohorts have examined the relations between radon exposure and cancer mortality, focusing especially on lung cancer (14, 15). They have concluded that cumulative exposure to radon is associated with an increase in lung cancer mortality. Several studies have suggested that radon exposure might also induce other kinds of cancer, such as leukemia, non-Hodgkin's lymphoma, malignant melanoma, multiple myeloma, and cancers of the pancreas, stomach and kidney, as well as other non-malignant respiratory diseases (16-24). Nonetheless, the only significant positive association reported to date between cumulative radon exposure and any cause of mortality is for lung cancer.

The analysis of CSD risks related to radon exposure is a very new topic, and only a few studies have examined them in detail. Four studies reported a decrease $(23,25-27)$ in cardiovascular mortality among miners exposed to radon compared with the general population, while one study found similar risk (17). Conversely, elevated mortality from coronary heart disease and nonrheumatic circulatory diseases was found in a cohort of sulphide-ore miners exposed to radon (28) and in a Czech cohort of uranium miners (29). A significant positive joint effect of radon and arsenic on CSD mortality was found in a cohort of Chinese tin miners (30). The Newfoundland fluorspar miner cohort study also found an elevated death rate from coronary heart diseases among miners with high cumulative radon exposure, but this finding was based on a relatively small number of deaths and was not statistically significant (31). Reanalysis of this cohort with ten additional years of follow-up showed no association between radon exposure and mortality from either CSD, acute myocardial infarction, or CeVD (32). A recent report about a large German uranium miner cohort found no trend with increasing cumulative exposure to radon for the risk of death from CSD for either the heart disease or stroke subgroups (33). All these findings should be interpreted with caution because it is unclear whether they reflect real associations, random variations, or confounding.
In 2007, we updated the mortality data for a French cohort of uranium miners from 1946-1999, including five additional years of follow-up (34). The analysis confirmed an increased risk of lung cancer death associated with radon exposure, even at low levels of exposure. The aim of this current study was to examine the CSD mortality in this French cohort of uranium miners and evaluate the possibility of an association with radon exposure. We consider the risk of death from CSD as a whole and from two subgroups: ischemic heart disease (IHD) and CeVD.

\section{Methods}

\section{Cohort description and follow-up}

Previous publications have described the development of a French cohort of uranium miners $(26,34,35)$. The cohort comprised 5086 individuals. It included all males employed as uranium miners in the CEA-COGEMA group [the French Atomic Energy Commission (CEA) and the COmpagnie GEnérale des MAtières Nucléaires (COGEMA today known as AREVA NC) were in charge of uranium exploitation in France] for $\geq 1$ year between 1946-1990. The follow-up began in 1946 and ran through 31 December 1999. The date of cohort entry for each individual was defined as the date of first employment as a uranium miner plus one year. Vital status was obtained from the French national vital status registry and classified into four categories: (i) dead, (ii) alive, (iii) alive at 85 years (age limit of follow-up), and (iv) lost to follow-up. Cause of death information came from the French national mortality database (direct or initial cause of death, as well as two antecedent causes or morbid conditions that contributed to death), supplemented by information from the COGEMA occupational medicine department. Causes of death were coded according to the International Classification of Disease (ICD) - the $8^{\text {th }}$ revision for deaths before 1979 and the $9^{\text {th }}$ revision for subsequent deaths.

\section{Cause of death from circulatory system diseases}

The overall group of deaths due to CSD corresponds to ICD 8 codes "390-458" and ICD 9 codes "390-459". IHD corresponds to ICD 8 and 9 codes " $410-414$ " and CeVD to ICD 8 and 9 codes " $430-438$ ".

\section{Place and type of work}

The French uranium mines were located in four regions and were active over different periods: Limousin (19461995), Vendée (1949-1991), Forez (1946-1978), and Hérault (1978-1997). The worksite was recorded every year for each miner. For this analysis, each miner was 
classified at a single mine location (ie, the one where he worked the most years). The cohort included underground and open-pit miners, and miners working both above and below the surface. The miners' working histories were reconstructed from their occupational records.

Based on information about the type of job they did, we classified physical activity for each miner and every year as "low", "intermediate", or "hard". Low physical activity corresponded to administrative, driving, or laboratory work; intermediate activity related to work in the mines not directly involving extraction, such as being an electrician or timberman. Until the end of the 1970 s, before the implementation of intensive mechanization, drillers and hewers performed hard physical labor. For this analysis, physical activity was classified on a 4-point scale according to the number of years of hard physical labor $(0=$ no hard physical activity; $1=0-4$ years; $2=5-14$ years; and $3=\geq 15$ years). This information was reconstructed for 5066 uranium miners.

\section{Radon exposure}

Exposure to radon and its radioactive decay products was estimated individually for each year of employment. The methods of measurement changed over time (34). For the years before 1956, annual individual exposure was retrospectively reconstructed by a group of experts, from environmental measurements in mine air and information about the place and duration of work. From 1956-1982, exposure was estimated from individual records. Exposure for each miner was routinely recorded each month in files, based on measurements of ambient radon gas concentrations at worksites and adjusted for time worked. Since 1983, radon exposure has been measured by individual dosimeters that determine the potential alpha energy of radon decay products. The exposure unit used for radon and decay products is the working-level month (WLM). The WL is defined as the concentration of short-lived radon daughters per litre of air that gives rise to $1.3 \times 10 \mathrm{e} 5 \mathrm{MeV}$ of alpha energy after complete decay. One WLM of cumulative exposure corresponds to exposure to $1 \mathrm{WL}$ for 1 month $(170 \mathrm{~h})$ and is equivalent to $3.5 \mathrm{~mJ} . \mathrm{h} . \mathrm{m}^{-3}$.

In 1956, large-scale ventilation was introduced in French mines, reducing radon exposure considerably. Mean annual exposure before 1956 was 21.3 WLM; after 1956, it was 1.7 WLM.

\section{Statistical analysis}

For each miner, the number of person-years was calculated as the time between entry into and exit from the cohort. Person-years were distributed into a 5-dimensional table: (i) attained age (10 categories), (ii) calendar year (11 categories), (iii) duration of employment (8 categories), (iv) age at first employment ( 8 categories), and (v) cumulative radon (6 categories). The expected numbers of deaths were calculated by multiplying person-years by the appropriate cause-specific mortality rates. National mortality rates for the general population of French men were used as reference rates. We assessed risk of death for cohort members using standardized mortality ratios (SMR) and estimated relative risks (RR) with an internal reference group of the 953 miners not exposed to radon. We calculated the $95 \%$ confidence intervals $(95 \% \mathrm{CI})$ for the SMR and RR using Byar's and Wald's approximations, respectively (36).

We tested trends in SMR and RR according to cumulative radon exposure and duration of employment using Mantel's trend tests. The risk of death was estimated for categories of cumulative radon exposure $(0,0-10$, $10-50,50-100,100-200$ and $\geq 200$ WLM and employment duration $(1-5,5-10,10-15,15-20,20-25,25-30$, $30-35$ and $\geq 35$ years). A 5-year lag time was applied for cumulative radon exposure to take into account a minimum latent period between exposure and effect. No lag time was applied to the duration of employment.

The exposure-risk relation was estimated with a linear excess relative risk (ERR) model, where the $\mathrm{RR}=1+\beta \mathrm{w}$, where $\mathrm{w}=$ cumulative radon exposure lagged by 5 years (in WLM) and $\beta=$ estimated ERR per 100 WLM.

We estimated the ERR with both external and internal regression analyses. For the external analyses, the baseline risk was assumed to be proportional to the expected number of deaths derived from national mortality rates. For the internal analyses, the baseline risk was stratified by calendar year and attained age. Poisson regression was used to fit these models. We used SAS (SAS Institute, Cary NC, USA) and EPICURE (HiroSoft International Corporation, Seattle, WA, USA) software for statistical analyses and applied the AMFIT module of EPICURE to calculate maximum likelihood parameter estimates, likelihood-based $\mathrm{CI}$ and likelihood ratio tests.

Sensitivity analyses also applied 10-, 20-, and 30year lag times. Additional stratification for two timeindependent variables was also considered, namely, hard physical activity and mine location.

\section{Results}

\section{Cohort characteristics}

Table 1 summarizes the cohort's characteristics. The study included 5086 subjects who contributed 153063 person-years of follow-up. Overall, 3492 (70\%) miners were still alive at the end of the follow-up, 1467 (28.8\%) were deceased, $66(1.3 \%)$ were lost to follow-up and $61(1.2 \%)$ were $\geq 85$ years. Miners started working at a mean age of 27.8 years and worked for an average 
duration of 16.4 years. The follow-up was relatively long with a mean duration of 30.1 years. Of 5086 miners, 4133 were exposed to radon, and the average cumulative exposure was 36.6 WLM. Mean age at first radon exposure was 29.1 years, and the average duration of exposure was 11.8 years.

\section{All-cause and all-cancer mortality}

The SMR and RR for all causes were not statistically significant in this cohort (results not shown). The SMR for all cancers was significantly $>1$ and significantly $<1$ for all causes excluding cancers and external causes. The RR was not statistically significant for either. A

Table 1. Characteristics of the French cohort of uranium miners. [WLM = working level month]

\begin{tabular}{|c|c|c|c|c|}
\hline & \multicolumn{4}{|c|}{ All subjects ( $N=5086)$} \\
\hline & Number & Percentage & Mean & Range \\
\hline \multicolumn{5}{|l|}{ General characteristics } \\
\hline Number of subjects & 5086 & 100 & . & .. \\
\hline Number of person-years & 153063 & 100 & . & .. \\
\hline Number of deaths & 1467 & 28.8 & . & .. \\
\hline Age at first employment (years) & . & .. & 27.8 & $15.0-67.4$ \\
\hline Duration of employment (years) & . & .. & 16.4 & $1.0-54.0$ \\
\hline Duration of follow-up (years) & - & .. & 30.1 & $0.1-53.7$ \\
\hline \multicolumn{5}{|l|}{ Radon exposure characteristics ${ }^{a}$} \\
\hline Age at first exposure (years) & . & .. & 29.1 & $15.0-63.0$ \\
\hline Cumulative exposure (WLM) & . & .. & 36.6 & $0.1-960.1$ \\
\hline Duration of exposure (years) & . & .. & 11.8 & $1.0-37.0$ \\
\hline Annual exposure <1956 (WLM) & . & .. & 21.3 & $0.1-99.0$ \\
\hline Annual exposure $\geq 1956$ (WLM) & . & .. & 1.7 & $0.1-15.3$ \\
\hline
\end{tabular}

a Only among radon-exposed miners (4133 miners). significant decreasing trend according to duration of employment was observed for overall mortality and all causes of death excluding cancers and external causes. Significant positive trends were observed according to cumulative radon exposure for overall and all-cancer mortality and for mortality from all causes excluding cancers and external causes. Similar results were observed for SMR and RR, except for deaths from all causes excluding cancers and external causes, which showed no significant trend according to cumulative radon exposure for the internal reference.

\section{Mortality from circulatory system diseases}

Table 2 shows the SMR and RR for different causes of CSD mortality. Overall it did not differ between miners and the French male population. Neither SMR nor RR differed significantly from 1 for CSD [ $\mathrm{N}=319$, SMR 0.93 (95\% CI 0.83-1.04) and RR 0.92 (95\% CI $0.72-1.19)]$. This was also true for the subgroups with IHD [N=125, SMR 0.94 (95\% CI 0.78-1.12) and RR 0.90 (95\% CI 0.60-1.35)] and CeVD [N=80, SMR 1.00 (95\% CI 0.79-1.24) and RR 1.39 (95\% CI 0.81-2.38)]. SMR and RR values were similar in both subgroups.

Trends with cumulative radon exposure were tested for all deaths from CSD, IHD and CeVD (table 2). The results for SMR and RR were similar for all three groups. Significant decreasing trends were observed according to the duration of employment for all CSD and IHD. Only CeVD showed a positive trend with cumulative radon exposure.

After we observed this significant positive trend for $\mathrm{CeVD}$ according to cumulative radon exposure, we conducted complementary detailed analyses of variations of mortality. Variations in CeVD mortality were

Table 2. Standardized mortality ratios (SMR) and relative risk (RR) of death from diseases of the circulatory system and trend tests with duration of employment and cumulative radon exposure. [95\% $\mathrm{Cl}=95 \%$ confidence interval]

\begin{tabular}{|c|c|c|c|c|c|c|c|c|c|c|}
\hline \multirow[t]{3}{*}{ Cause of death ${ }^{\mathrm{a}}$} & \multicolumn{2}{|c|}{ Observed deaths } & \multicolumn{4}{|c|}{ External reference } & \multicolumn{4}{|c|}{ Internal reference } \\
\hline & \multirow[t]{2}{*}{$\mathrm{N}$} & \multirow[t]{2}{*}{$\%$} & \multirow[t]{2}{*}{$\mathrm{SMR}^{\mathrm{b}}$} & \multirow[t]{2}{*}{$95 \% \mathrm{Cl}$} & \multicolumn{2}{|c|}{ Trend test } & \multirow[t]{2}{*}{$\mathrm{RR}^{\mathrm{c}}$} & \multirow[t]{2}{*}{$95 \% \mathrm{Cl}$} & \multicolumn{2}{|c|}{ Trend test } \\
\hline & & & & & $\begin{array}{c}\text { Duration } \\
\text { of } \\
\text { employment } \\
(P \text {-value })^{d}\end{array}$ & 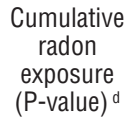 & & & $\begin{array}{c}\text { Duration } \\
\text { of } \\
\text { employment } \\
(P \text {-value })^{d}\end{array}$ & $\begin{array}{l}\text { Cumulative } \\
\text { radon } \\
\text { t exposure } \\
\text { (P-value) }^{d}\end{array}$ \\
\hline All circulatory system diseases (390-459) & 319 & 6.2 & 0.93 & $0.83-1.04$ & $0.02^{\mathrm{e}}$ & 0.18 & 0.92 & $0.72-1.19$ & $0.001^{\mathrm{e}}$ & 0.37 \\
\hline Ischemic heart diseases (410-414) & 125 & 2.4 & 0.94 & $0.78-1.12$ & $0.03^{e}$ & $>0.50$ & 0.90 & $0.60-1.35$ & $0.02^{e}$ & $>0.50$ \\
\hline Cerebrovascular diseases (430-438) & 80 & 1.6 & 1.00 & $0.79-1.24$ & $>0.50$ & $0.02^{\dagger}$ & 1.39 & $0.81-2.38$ & $>0.50$ & $0.03^{f}$ \\
\hline
\end{tabular}

\footnotetext{
a The code according to the International Classification of Diseases, $9^{\text {th }}$ revision in parentheses.

b The reference group is the French male population.

c The reference category is made of non-radon-exposed miners.

d Degree of significance of a Mantel's trend test.

e Negative significant trend.

f Positive significant trend.
} 
examined by calendar period, attained age, duration of employment, and age at time of first employment (figure 1). Figure 1A shows that, up to 1991, the number of expected deaths from CeVD is higher than that of observed deaths; after 1992, both become similar. Figure 1B shows the global lack of difference between the numbers of observed and expected deaths, except for an excess, not statistically significant, of observed deaths among the miners aged 65-70 years. A specific pattern of CeVD mortality linked with the duration of employment cannot be deduced from figure 1C. Finally, figure 1D shows a statistically non-significant excess of observed deaths for miners who started working at the age of 25-30 years.

\section{Radon exposure and risk of circulatory system disease}

Table 3 shows the estimated ERR per 100 WLM for the three categories of CSD mortality associated with cumulative radon exposure for both the internal and external Poisson regression analyses. Results were consistent with those from the trend tests. Estimated ERR was significant only for CeVD and was similar for the internal [ERR per $100 \mathrm{WLM} 0.44$ (95\% CI 0.04-1.16)] and external [ERR per 100 WLM 0.49 (95\% CI $0.07-$ 1.23)] regression analyses. For IHD, lower confidence bounds could not be estimated. SMR by categories of radon exposure together with a linear ERR model for CeVD mortality are presented in figure 2 . Above 50 WLM, the risk of CeVD mortality increased, albeit not significantly.

The main analysis used a 5-year lag period. In the sensitivity analysis, ERR per 100 WLM was estimated with three different lag periods: 10, 20, and 30 years, with the internal regression. This did not change the results, which remained non-significant for overall CSD and IHD for the different lag analyses. For the CeVD subgroup, however, the estimated ERR per 100 WLM remained significant and increased with the lag period (table 4).

The second part of the sensitivity analysis stratified the workers according to two supplementary variables: hard physical activity and mine location (reflecting the geological characteristics of the mine). Both stratifications had no impact on the estimated ERR per 100 WLM for overall CSD and IHD. For CeVD, the ERR per 100 WLM estimated after stratification for hard physical activity was not significant [ERR per 100 WLM 0.21 (95\% CI -*-1.01), P=0.29]. It was, however, significant after stratification for mine location [ERR per 100 WLM 0.54 (95\% CI 0.07-1.41), $\mathrm{P}=0.015$ ] and almost significant after stratification for both hard physical activity and mine location [ERR per $100 \mathrm{WLM}$ $0.51(95 \% \mathrm{CI}-*-1.7), \mathrm{P}=0.058]$.
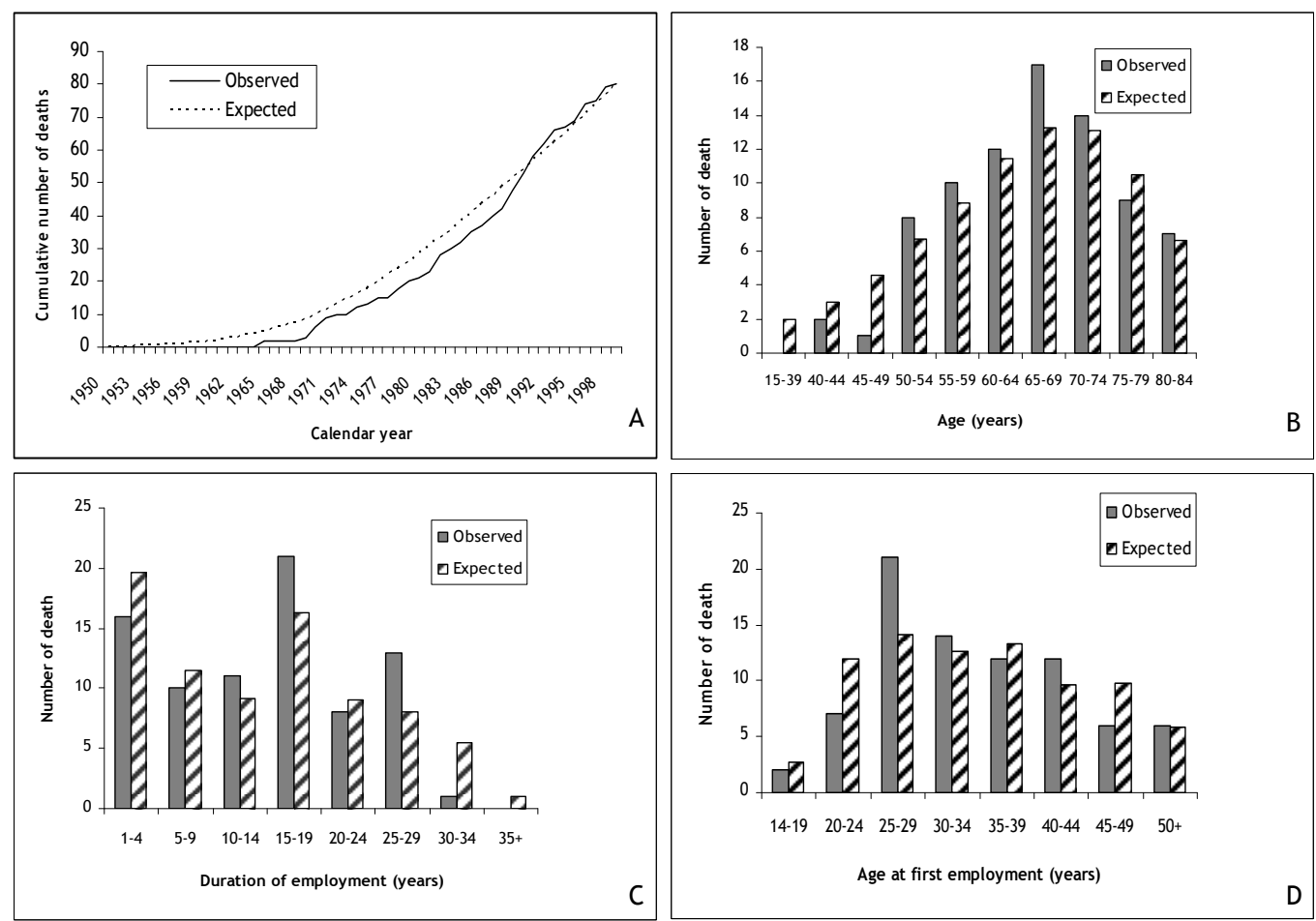

Figure 1. Variations of observed and expected numbers of cerebrovascular deaths from 1946-1999 in the French cohort of uranium miners with: (A) calendar year; (B) attained age; (C) duration of employment; and (D) age at first employment. 
Table 3. Risk of mortality from overall circulatory system, ischemic, and cerebrovascular diseases associated with cumulative radon exposure in the French cohort of uranium miners. [RR $=$ relative risk, $\mathrm{WLM}=$ working level month, $95 \% \mathrm{Cl}=95 \%$ confidence interval]

\begin{tabular}{lcc}
\hline $\begin{array}{l}\text { Regression } \\
\text { type }\end{array}$ & Intercept & Excessive \\
& per RR \\
& 100 WLM & \\
& & \\
\hline
\end{tabular}

\begin{tabular}{lrrr}
\hline All circulatory system diseases & & & \\
External $^{\mathrm{a}}$ & 0.89 & 0.102 & $-0.04-0.30$ \\
Internal $^{\mathrm{b}}$ & - & 0.064 & $-0.06-0.25$ \\
Ischemic heart disease & & & \\
$\quad$ External & 0.93 & 0.013 & $-\mathrm{c}-0.28$ \\
$\quad$ Internal & - & 0.007 & $-\mathrm{c}-0.27$ \\
Cerebrovascular disease & & & \\
$\quad$ External & 0.83 & 0.492 & $0.07-1.23$ \\
Internal & - & 0.437 & $0.04-1.16$ \\
\hline
\end{tabular}

a The reference group is the French male population.

${ }^{\mathrm{b}}$ The reference group consists of non-radon-exposed miners.

c Not estimated.

\section{Discussion}

This first detailed analysis of CSD mortality in the French uranium miner cohort found no excess risk for CSD, IHD, or CeVD mortality. We did, however, observe a significant increase in CeVD mortality risk with cumulative radon exposure that had not been shown in the earlier analyses. This relation was confirmed with other lag intervals. Risks of CSD and IHD were not associated with cumulative radon exposure.

\section{Characteristics of the French cohort}

This cohort of French uranium miners was characterized by its long follow-up and very low levels of cumulative radon exposure. Its high quality of exposure assessment was also notable, especially after 1955 , thanks to individual dosimetric recording. Moreover, the high quality

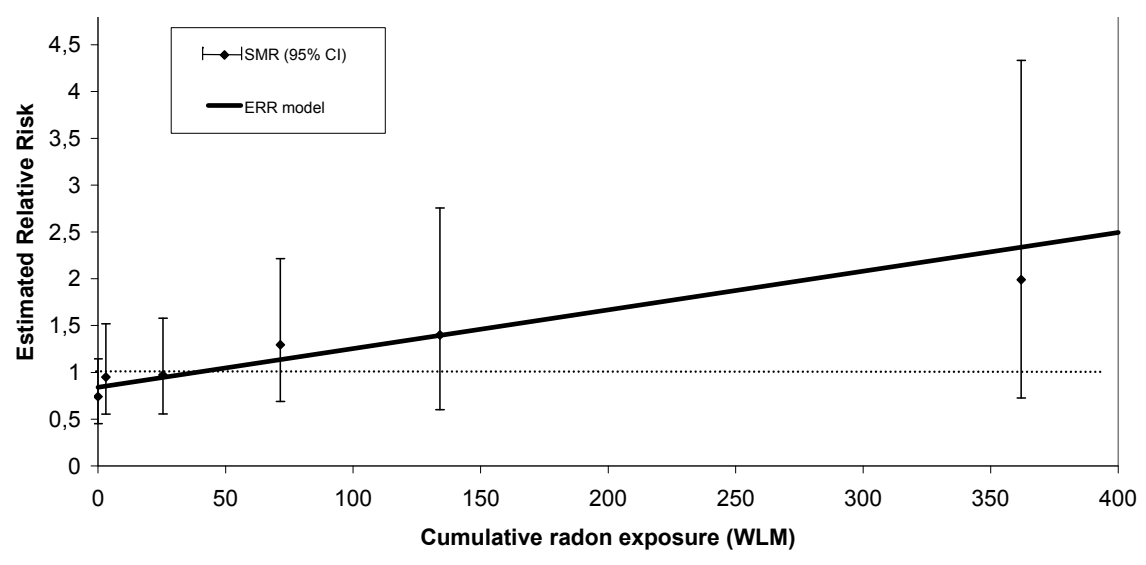

Figure 2. Cerebrovascular mortality risk associated with cumulative radon exposure in the French cohort of uranium miners, 1946-1999. [SMR=standardized mortality ratio, $95 \% \mathrm{Cl}=95 \%$ confidence interval, ERR model=linear excess relative risk model with external reference, WLM=working level month]

Table 4. Excess relative risk associated with cumulative radon exposure estimated with 5-, 10-, 20-, and 30-year lag periods. [ERR=excess relative risk, WLM=working level month, $95 \% \mathrm{Cl}=95 \%$ confidence interval]

\begin{tabular}{|c|c|c|c|c|c|c|c|c|}
\hline \multirow[t]{2}{*}{ Cause of death } & \multicolumn{2}{|c|}{ 5-year lag } & \multicolumn{2}{|c|}{ 10-year lag } & \multicolumn{2}{|c|}{ 20-year lag } & \multicolumn{2}{|c|}{ 30-year lag } \\
\hline & $\begin{array}{c}\text { ERR per } \\
100 \text { WLM }\end{array}$ & $95 \% \mathrm{Cl}^{\mathrm{a}}$ & $\begin{array}{c}\text { ERR per } \\
100 \text { WLM }\end{array}$ & $95 \% \mathrm{Cl}^{\mathrm{a}}$ & $\begin{array}{c}\text { ERR per } \\
100 \text { WLM }\end{array}$ & $95 \% \mathrm{Cl}^{\mathrm{a}}$ & $\begin{array}{c}\text { ERR per } \\
100 \text { WLM }\end{array}$ & $95 \% \mathrm{Cl}^{\mathrm{a}}$ \\
\hline All circulatory system diseases & 0.064 & $-0.06-0.25$ & 0.067 & $-0.06-0.25$ & 0.065 & $-0.07-0.26$ & 0.08 & $-0.083-0.34$ \\
\hline Ischemic heart disease & 0.007 & $-b-0.27$ & 0.008 & $-b-0.28$ & -0.025 & $-b-0.25$ & -0.126 & $-b$ \\
\hline Cerebrovascular disease & 0.44 & $0.037-1.16$ & $0.46^{c}$ & $0.047-1.21$ & $0.53^{d}$ & $0.066-1.37$ & $0.85^{\mathrm{e}}$ & $0.17-2.17$ \\
\hline
\end{tabular}

a Obtained from internal regression

${ }^{\mathrm{b}}$ Not estimated; degree of significance of a two-sided likelihood ratio test comparing the models with and without radon exposure.

c $P=0.021$.

d $P=0.016$

e $P=0.005$. 
of the follow-up was evident by the very low percentage of miners lost to follow-up $(1.3 \%, \mathrm{~N}=63)$ and the low percentage of deaths for which the cause of death is missing $(4 \%, \mathrm{~N}=56)$.

\section{Definition of circulatory system disease subgroups}

In this analysis, we used subgroups of CSD similar to those of other studies [ie, IHD and $\operatorname{CeVD}(8,10,30,32$, $33)]$. It has been recently suggested that IHD might be a particularly sensitive endpoint for radiation exposure to the heart arteries (37). In the French cohort, the IHD subgroup represented almost $40 \%$ of the CSD deaths (ie, 125 dead miners). Several studies have shown an increased risk of $\mathrm{CeVD}$ associated with external ionizing radiation $(6,10)$. In the French cohort, this subgroup accounted for 80 dead miners.

CSD are particularly subject to misclassification bias (38). A European project indicated, however, that the French national cause-of-death statistics from cardiovascular diseases are reliable and that when the subcategories are adequately grouped (such as IHD and CeVD), the published mortality data can be considered to be sufficient for epidemiological purposes (39). We therefore chose to focus our analysis on only two subgroups, with a sufficient number of deaths. The use of different sources of information for causes of death may also lead to a misclassification bias. Information for our cohort came from two sources. After 1968, the French national mortality database provided causes for $97.4 \%$ of the deaths, while information from the occupational medical department was used only as a complementary source. To avoid this potential misclassification bias, mortality was analyzed for miners who died between 1968-1999; this analysis confirmed the results from the main analysis and thus the absence of any misclassification bias.

Misclassification bias might also have stemmed from a classification error between the initial and associated causes of death. We therefore performed mortality analyses that took into account the initial and associated CeVD causes of death. These results also confirmed the absence of misclassification bias.

\section{Mortality from circulatory system diseases}

Our analysis did not show any excess risk of overall CSD, IHD, or CeVD mortality among the French uranium miners. These results confirmed those of previous analyses of this cohort (26). Similarly, other studies of radon-exposed miners have found no excess risk of CSD mortality $(17,33,40)$. On the other hand, other studies of radon-exposed miners have reached different and inconsistent results for detailed CSD mortality. A significant excess mortality risk for coronary heart disease was found in a cohort of sulphide-ore miners exposed to radon [observed $=44$, expected $=22.1$ (28)]. Another elevated SMR for non-rheumatic circulatory disease was found in a Czech cohort of uranium miners [SMR 1.16 (95\% CI 1.08-1.25) (29)]. Still other studies report significantly reduced risks of circulatory and coronary mortality. In a cohort study of Newfoundland fluorspar miners, the SMR for coronary heart disease was 0.86 (95\% CI 0.74-0.89) (32). In a Colorado Plateau uranium miner cohort, the SMR for heart disease was $0.6(95 \%$ CI $0.4-0.6)$ and $0.4(95 \%$ CI $0.2-0.7)$ for circulatory diseases (27). The significantly lower death rates in those studies reflect the healthy-worker effect. To evaluate this effect, likely to be large for cardiovascular diseases (38), we considered an internal reference population. In our cohort, both the SMR and RR were similar for CSD and IHD. Nevertheless, the SMR calculated for CeVD was equal to $1.00(95 \%$ CI $0.8-1.2)$ while the RR was equal to 1.39 (95\% CI $0.8-2.4$ ), possibly because of the healthy-worker effect. Trends with duration of employment also show the existence of this effect. Indeed, in most industries, individuals employed for very short periods of time have substantially higher mortality rates than those employed for longer periods (41). Detailed analyses showed that the decreasing trend for CeVD can be partly explained by a significant deficit of mortality in the category of workers employed for 30-35 years.

\section{Radon exposure and risk of circulatory system diseases}

Our analysis did not find an association between cumulative radon exposure and either overall CSD or IHD mortality. A significant positive trend according to cumulative radon exposure was nonetheless found for the CeVD subgroup. This result was confirmed by both the internal [ERR per 100 WLM $0.44(95 \%$ CI 0.04 1.16)] and external [ERR per $100 \mathrm{WLM} 0.49(95 \% \mathrm{CI}$ $0.07-1.23)]$ regression analyses. The consistency of the significant trend and ERR obtained with both internal and external reference groups consolidated the validity of the association observed between cumulative radon exposure and CeVD mortality. We controlled for the effects of age and calendar period in all our analyses. Nevertheless, we were unable to test for a potential modifying effect of attained age or time since exposure as has been seen for lung cancer risk (42). Additional analyses were performed to determine the range of exposure for which a significant relation between radon exposure and CeVD risk was observed. Analyses showed that the observed relation was no more significant when the exposure range was restricted to 0-275 WLM.

Several studies have reported analyses about the relation between radon exposure and CSD. Xuan et al (30) reported significant positive trends for coronary heart disease and CeVD in the Chinese tin cohort, but 
they could not separate the effects of radon and arsenic on cardiovascular mortality. They did not demonstrate the existence of an exposure-risk relation. In the Czech cohort of uranium miners, a statistically non-significant, negative trend with cumulative radon exposure was observed for non-rheumatic circulatory diseases (29). In the large German cohort, there was no trend for the risk of CSD with increasing cumulative exposure for either the heart disease or stroke subgroups (33). The Newfoundland cohort study of fluorspar miners found elevated death rates from coronary heart diseases among the miners with higher cumulative radon exposure, but this finding was based on a relatively small number of deaths and was not statistically significant [RR 1.46 (95\% CI 0.77-2.75)] for miners with cumulative radon exposure $\geq 1000$ WLM (31). When mortality data were updated for an additional 10 years, through the end of 2001, no relation was found between cumulative radon exposure and mortality from CSD, acute myocardial infarction, or CeVD (32).

The briefness of the average duration of radon exposure (5.6 years) may explain this finding, because the pathophysiological processes that lead to death in circulatory system diseases are known to be long.

To our knowledge, no other study of miners has reported such an association between radon exposure and CeVD risk as observed in our study. This could be a chance finding. But on the other hand, the characteristics of this French cohort (ie, good quality of exposure assessment, long duration of exposure, long duration of follow-up) may play a role in this discrepancy. In some studies, the briefness of the average duration of radon exposure may explain the absence of an association if the pathophysiological processes that lead to death in circulatory system diseases is very long. In addition, we performed a sensitivity analysis that showed an increase in the estimated ERR per 100 WLM with the lag period in our cohort. The estimated ERR was twice as high with a 30-year lag time [ERR per 100 WLM 0.85 (95\% CI $0.17-2.17)$ ] than with a 5 -year lag time [ERR per 100 WLM 0.44 (95\% CI 0.037-1.16)]. This result may be a supplementary argument in favor of a link between $\mathrm{CeVD}$ and cumulative radon exposure, if as pointed out above, circulatory diseases develop over a long period before becoming lethal.

\section{Potential biological mechanism}

Several authors have suggested that small amounts of alpha radiation in the arterial wall may cause subtle injuries to arterial structures and thus contribute to the development of atherosclerosis and consequently CSD $(31,43)$. Much research has been initiated to investigate the possible effects of low doses of radiation on the arteries, especially in the framework of ongoing
European research projects. Several mechanisms have been proposed $(2,12,13)$. Three main hypotheses could be mentioned. The first one is the inflammation theory; it implies an over-expression of pro-inflammatory molecules, such as interleukin 6 and C-reactive protein, which could lead to a inflammatory process associated with endothelial damage and dysfunction, responsible for the development of atherosclerosis. The second hypothesis is called the microvasculature theory; it is based on the vascular radiation-induced damage (such as fibrosis or thickening of the coronary arteries) that is observed after high doses of irradiation. The third is the monoclonal theory, in which a genetic instability induced by radiation may be responsible for the transformation and monoclonal proliferation of smooth muscle cells and the development of atheromatous plaques (2). In a recent paper, Little et al (13) proposed an arguably more plausible mechanism for fractionated low-dose effects based on monocyte-cell killing in the intima.

Nevertheless, for the time being, no potential mechanism seems to be sufficient to explain a causal relationship between radiation exposure and CSD risk, and mechanisms may even be different for coronary arteries, the myocardium, or peripheral arteries. To our knowledge, no specific mechanism is suspected for cerebral vessels. But in the case of CeVD, we could also hypothesize that the association is not only due to a direct effect, but rather some modification of CeVD risk factors, such as hypertension or cholesterolemia. In this regard, it is interesting to mention that among atomicbomb survivors, in addition to the association observed with a stroke (6), a dose-response relationship was also observed with hypertension (44) and cholesterol level (45). In that case, the vascular epithelium may not be the only organ pertaining to an association between $\mathrm{CeVD}$ risk and radiation exposure; several organs involved in the regulation of these parameters, such as the kidney or the liver, may also be relevant.

Among miners, radon exposure is expressed in WLM. This unit corresponds to the potential alpha energy due to radon decay products, and it is considered to be a good indicator of the dose delivered to the lung after inhalation. Nevertheless, WLM is not an organdose unit, and the dose absorbed by other organs may be very different from that of the lung. The US National Research Council report on the health effects of exposure to radon stated that, according to dosimetric estimates, doses to the blood and arteries are less than $1 \%$ of those absorbed by the lung (15). More recently, some calculations of doses to specific organs were recently developed in the framework of the European Alpha-Risk collaborative research project (www.alpha-risk.org), based on a close collaboration between epidemiologists and dosimetrists. Doses to the red bone marrow, liver, and kidney were estimated (46). These calculations 
considered the multiple chronic exposures of the miners to radon gas and radon decay products, external gamma exposure, and incorporation of long-lived radionuclides due to inhalation of uranium ore dust. The estimated doses were indeed much lower than those delivered to the lungs, but they were in some instances not negligible. The relationship between CeVD risk and cumulated radon exposure observed in our study should therefore be cautiously considered with respect to the existence of a potential dose-risk relationship, but we think that an analysis of CSD risk on the basis of specific organ dose estimations constitutes a very interesting route of research for the future.

In summary, although our results show a significant relationship between CeVD risk and radon exposure, we must remain very careful about interpreting a potential causal association. This result has to be confirmed by other studies on miners, and more research is needed to explore the potential susceptibility of cerebral vessels to ionizing radiation.

\section{Other factors}

The appropriate interpretation of our results requires a discussion of the potential limitations of our study, including confounding risk factors.

Numerous risk factors for CSD have been identified, and our findings may be biased by our inability to adjust for their influence. Occupational factors that are associated with an increased risk of CSD include stress levels, shift work, noise, and temperature (47). Other risk factors include diabetes, obesity, cholesterol, triglyceride levels, and alcohol intake (48). Socioeconomic status has also been shown to be associated with cardiovascular disease risk. Unfortunately, data on these factors were not available in our cohort. In addition, tobacco consumption is known to be an important risk factor for CSD. Information on smoking habits was not available individually for all miners from the French cohort. The Newfoundland uranium cohort did not find that smoking status confounded the risk estimated for coronary heart disease (32). It would be interesting in future studies to adjust results for covariates such as tobacco, blood pressure, or body mass index. Collection of such information from medical archives may be possible in the future from a nested case-control approach.

The working conditions and some pollutants present in the mine atmosphere may also play a role in the CSD risk of miners. In previous analyses of our cohort, hard physical activity and mine type appeared as modifying factors of the radon-lung cancer risk relationship (42). Accordingly, we took these factors into account in this analysis. After adjusting for hard physical activity, the estimated ERR was not significant, thus suggesting an interaction between hard physical activity and cumulative radon exposure for CeVD risk. In addition to radon, miners were exposed to other pollutants present in the mines' atmosphere. These pollutants included other radiological exposures, such as gamma rays, uranium ore dust, fine dust, temperature, or diesel exhausts among others. Individual information about these pollutants is rarely available, and their potential impact is difficult to evaluate. Sjörgren's review (49) has suggested that particulate matter, and specially the ultrafine components of dust, might have effects on the development of IHD. Moreover, another study suggested that particulate matter may be responsible for changing red cell adhesiveness on lung endothelial cells (50). Exposure to fine dust, therefore, can possibly play a role in the risk for CSD and confound the estimated relationship between radon exposure and CeVD risk. But this exposure varies a lot in mines according to the ventilation and extraction method, and individual exposure estimates are generally not available in miner cohorts. Other hazardous substances present in the underground environment, such as external gamma radiation, may also have contributed to modifying the exposure-risk relation. Several studies, such as the lifespan cohort study, observed an increased risk of CeVD associated with external ionizing radiation $(5,6)$. However, it is important to note that the exposure of atomic-bomb survivors was very different from that of miners: in the former, exposure was delivered over a very short time interval whereas the dose was cumulated over the whole working life among the latter. In other studies of miners, no association have been observed between CeVD and cumulative gamma radiation $(29,33)$. Miners were also exposed to ore dust, which could lead to non-negligible doses to specific organs due to the incorporation of long-lived radionuclides. Gamma and uranium ore dust exposures were reconstructed in the French cohort after 1956. In the future, statistical models should be developed to take into account simultaneously the effects of radon, gamma, and ore dust exposure on CeVD risk.

\section{Concluding remarks}

Results about the potential relationship between ionizing radiation and CSD in studies of miners have been few and inconclusive. Studies from Canadian and German miner cohorts did not support an association between circulatory diseases and exposure to radon $(32,33)$. Like these studies, we did not find any excess of cardiovascular mortality. But our results suggested the existence of an association between CeVD mortality and cumulative radon exposure. This is the first uranium miner cohort study in which such an association has been observed. One important advantage of this French cohort is the quality of the radon exposure assessment. Hard physical activity has been identified as a potential modifying 
factor of the exposure-risk relation. However, our findings should be interpreted with caution as possible confounding by several CSD risk factors could not be adequately assessed.

Further extension of this work may be done in the future by integrating information on smoking and other biological indicators (considering exposures other than radon in uranium mines) and calculating organ dosage on the basis of what was initiated in the framework of the European $\alpha$-RISK collaborative research project.

\section{Acknowledgements}

This work was partly supported by AREVA NC, in the framework of a bilateral IRSN-AREVA NC agreement, and by the European Commission under the $6^{\text {th }}$ Framework Program for Research and Technological Development (“Alpha-Risk project”, contract: FI6R 516483). The authors are grateful to: G Lauret, JC Hyvernaud, C Martinet from AREVA NC, S Bernhard from Algade and JC Filloux from L'Association Interprofessionnelle pour la Santé au Travail (AIST 87) for their help in data collection using the mines' archives. We also acknowledge G Antonini for her contribution to this study.

\section{References}

1. Sheehan J. Foam cell plaques in the intima of irradiated small arteries. Arch Pathol. 1944;37:297-308.

2. United Nations Scientific Committee on the Effects of Atomic Radiation (UNSCEAR). Effects of ionising radiation: epidemiological evaluation of cardiovascular disease and other non-cancer diseases following radiation exposure. New York (NY): United Nations; 2006. Report, volume 1, Scientific Annex B, p 323-69.

3. Rutqvist L, Lax I, Fornander T, Johannson H. Cardiovascular mortality in a randomized trial of adjuvant radiation therapy versus surgery alone in primary breast cancer. Int $\mathrm{J}$ Radiat Oncol Biol Phys. 1992;22(5):887-96.

4. Fajardo LF, Stewart JR, Cohn KE. Morphology of radiationinduced heart disease. Arch Pathol. 1968;86(5):512-9.

5. Gaya A, Ashford R. Cardiac complications of radiation therapy. Clin Oncol (R Coll Radiol). 2005;17:153-9.

6. Preston D, Shimizu Y, Pierce D, Suyama A, Mabuchi K. Studies of mortality of atomic bomb survivors, report 13: solid cancer and noncancer disease mortality: 1950-1997. Radiat Res. 2003;160:381-407.

7. McGale P, Darby SC. Low doses of ionizing radiation and circulatory diseases: a systematic review of the published epidemiological evidence. Radiat Res. 2005;163(3):247-57.

8. Hauptmann M, Mohan AK, Doody MM, Linet MS, Mabuchi
K. Mortality from diseases of the circulatory system in radiologic technologists in the United States. Am J Epidemiol. 2003;157(3):239-48.

9. Vrijheid M, Cardis E, Ashmore P, Auvinen A, Bae JM, Engels $\mathrm{H}$, et al. Mortality from diseases other than cancer following low doses of ionizing radiation: results from the 15Country Study of nuclear industry workers. Int J Epidemiol. 2007;36(5):1126-35.

10. Ivanov VK, Maksioutov MA, Chekin SY, Petrov AV, Biryukov AP, Kruglova ZG, et al. The risk of radiation-induced cerebrovascular disease in Chernobyl emergency workers. Health Phys. 2006;90(3):199-207.

11. Metz-Flamant C, Bonaventure A, Milliat F, Tirmarche M, Laurier D, Bernier MO. Irradiations à faibles doses et risque de pathologie cardiovasculaire: revue des études épidémiologiques. Rev Epidémiol Santé Publique. 2009;57(5):347-59.

12. Little MP, Tawn EJ, Tzoulaki I, Wakeford R, Hildebrandt $\mathrm{G}$, Paris F, et al. A systematic review of epidemiological associations between low and moderate doses of ionizing radiation and late cardiovascular effects, and their possible mechanisms. Radiat Res. 2008;169(1):99-109.

13. Little MP TE, Tzoulaki I, Wakeford R, Hildebrandt G, Paris F, Tapio S, et al. Review and meta-analysis of epidemiological associations between low/moderate doses of ionizing radiation and circulatory disease risks, and their possible mechanisms. Radiat Environ Biophysics. 2009 Oct 28 [Epub ahead of print].

14. Lubin J. Radon and lung cancer risk: a joint analysis of 11 underground miners studies. Washington (DC): National Institutes of Health (NIH), National Cancer Institute; 1994. NIH publication number 94-3644.

15. National Research Council. Health effects of exposure to radon (BEIR VI). Washington (DC): National Academy Press; 1999.

16. Rericha V, Kulich M, Rericha R, Shore DL, Sandler DP. Incidence of leukemia, lymphoma, and multiple myeloma in Czech uranium miners: a case-cohort study. Environ Health Perspect. 2006;114(6):818-22.

17. Roscoe RJ. An update of mortality from all causes among white uranium miners from the Colorado Plateau Study Group. Am J Ind Med. 1997;31(2):211-22.

18. Darby S. Radon and cancers other than lung cancer in underground miners: a collaborative analysis of 11 studies. J Natl Cancer Inst. 1995;

19. Tomasek L, Darby SC, Swerdlow AJ, Placek V, Kunz E. Radon exposure and cancers other than lung cancer among uranium miners in West Bohemia. Lancet. 1993;341(8850):919-23.

20. Kusiak RA, Ritchie AC, Springer J, Muller J. Mortality from stomach cancer in Ontario miners. $\mathrm{Br} \mathrm{J}$ Ind Med. 1993;50(2):117-26.

21. Hodgson JT, Jones RD. Mortality of a cohort of tin miners 1941-86. Br J Ind Med. 1990;47(10):665-76.

22. Henshaw DL, Eatough JP, Richardson RB. Radon as a causative factor in induction of myeloid leukaemia and other cancers. Lancet. 1990;335(8696):1008-12.

23. Morrison H, Semenciw R, Mao Y, Wigle D. Cancer mortality 
among a group of fluorspar miners exposed to radon progeny. Am J Epidemiol. 1988;128:1266-75.

24. Radford EP, Renard KG. Lung cancer in Swedish iron miners exposed to low doses of radon daughters. N Engl J Med. 1984;310(23):1485-94.

25. Samet JM, Pathak DR, Morgan MV, Key CR, Valdivia AA, Lubin JH. Lung cancer mortality and exposure to radon progeny in a cohort of New Mexico underground uranium miners. Health Phys. 1991;61(6):745-52.

26. Tirmarche M, Raphalen A, Allin F, Chameaud J, Bredon P. Mortality of a cohort of French uranium miners exposed to relatively low radon concentrations. Br J Cancer. 1993;67(5):1090-7.

27. Roscoe RJ, Deddens JA, Salvan A, Schnorr TM. Mortality among Navajo uranium miners. Am J Public Health. 1995;85(4):535-40.

28. Ahlman K, Koskela RS, Kuikka P, Koponen M, Annanmäki M. Mortality among sulfamide ore miners. Am J Ind Med. 1991;19(5):603-17.

29. Tomasek L, Swerdlow AJ, Darby SC, Placek V, Kunz E. Mortality in uranium miners in west Bohemia: a long-term cohort study. Occup Environ Med. 1994;51(5):308-15.

30. Xuan XZ, Lubin JH, Li JY, Yang LF, Luo AS, Lan Y, et al. A cohort study in southern China of tin miners exposed to radon and radon decay products. Health Phys. 1993;64(2):120-31.

31. Villeneuve PJ, Morrison HI. Coronary heart disease mortality among Newfoundland fluorspar miners. Scand J Work Environ Health. 1997;23(3):221-6.

32. Villeneuve PJ, Lane RS, Morrison HI. Coronary heart disease mortality and radon exposure in the Newfoundland fluorspar miners' cohort, 1950-2001. Radiat Environ Biophys. 2007;46(3):291-6.

33. Kreuzer M, Kreisheimer M, Kandel M, Schnelzer M, Tschense A, Grosche B. Mortality from cardiovascular diseases in the German uranium miners cohort study, 1946-1998. Radiat Environ biophys. 2006;45(3):159-66.

34. Vacquier B, Caer S, Rogel A, Feurprier M, Tirmarche M, Luccioni C, et al. Mortality risk in the French cohort of uranium miners: extended follow-up 1946-1999. Occup Environ Med. 2008;65(9):597-604.

35. Laurier D, Tirmarche M, Mitton N, Valenty M, Richard P, Poveda S, et al. An update of cancer mortality among the French cohort of uranium miners: extended follow-up and new source of data for causes of death. Eur J Epidemiol. 2004;19(2):139-46.

36. Breslow NE, Day NE. Statistical methods in cancer research, vol 2: the design and analysis of cohort studies. Lyon: International Agency for Research on Cancer (IARC); 1987. IARC Scientific Publication, number 82, p 1-406.
37. Yamada M, Wong FL, Fujiwara S, Akahoshi M, Suzuki G. Noncancer disease incidence in atomic bomb survivors, 19581998. Radiat Res. 2004;161(6):622-32.

38. Ducimetiere P, Jougla E, Haas B, Montaye M, Ruidavets JB, Amouyel $\mathrm{P}$, et al. Coronary mortality in France according to data sources. Rev Epidemiol Santé Publique. 2006;54(5):453-61.

39. Institute National de la Santé et de la Recherche Médical (INSERM). Comparability and quality improvement of European causes of death statistics. Brussels: European Commission; 2001.

40. Veiga LH, Amaral EC, Colin D, Koifman S. A retrospective mortality study of workers exposed to radon in a Brazilian underground coal mine. Radiat Environ Biophys. 2006;45(2):125-34.

41. Baillargeon $\mathrm{J}$ Characteristics of the healthy worker effect. Occup Med. 2001;16(2):359-66.

42. Vacquier B, Rogel A, Leuraud K, Caer S, Acker A, Laurier D. Radon-associated lung cancer risk among French uranium miners: modifying factors of the exposure-risk relationship. Radiat Environ Biophys. 2009;48(1):1-9.

43. Elkeles A. Alpha ray activity in coronary artery disease. J Am Geriatr Soc. 1968;16:576-83.

44. Sasaki H, Wong FL, Yamada M, Kodama K. The effects of aging and radiation exposure on blood pressure levels of atomic bomb survivors. J Clin Epidemiol. 2002;55:974-81.

45. Wong FL, Yamada M, Sasaki H, Kodama K, Hosoda Y. Effects of radiation on the longitudinal trends of total serum cholesterol levels in the atomic bomb survivors. Radiat Res. 1999;151:736-46.

46. Marsh JW, Bessa Y, Birchall A, Blanchardon E, Hofmann W, Nosske D, et al. Dosimetric models used in the Alpha-Risk project to quantify exposure of uranium miners to radon gas and its progeny. Radiat Prot Dosimetry. 2008;130(1):101-6.

47. Kristensen TS. Cardiovascular diseases and the work enviromnent: a critical review of the epidemiologic litterature on nonchemical factors. Scand J Work Environ Heatlh. 1989;15(3):165-79.

48. Wilson PW. Established risk factors and coronary artery diseases: the Framingham Study. Am J Hypertens. 1994;7(2):7S-12S.

49. Sjögren B. Occupational exposure to dust: inflammation and ischaemic heart disease. Occup Environ Med. 1997;54:466-9.

50. Seaton A, Soutar A, Crawford V, Elton R, McNerlan S, Cherrie J, et al. Particulate air pollution and the blood. Thorax. 1999;54:1027-32.

Received for publication: 3 July 2009 